\title{
Therapeutic Drug Monitoring of Voriconazole: Comparison of Bioassay with High-Performance Liquid Chromatography
}

\author{
Parisa Badiee, ${ }^{1}$ Zahra Hashemizadeh, ${ }^{1,}$ and Hashem Montaseri ${ }^{2}$ \\ ${ }^{1}$ Prof. Alborzi Clinical Microbiology Research Center, Shiraz University of Medical Sciences, Shiraz, IR Iran \\ ${ }^{2}$ Department of Pharmaceutics, Faculty of Pharmacy, Shiraz University of Medical Sciences, Shiraz, IR Iran \\ "Corresponding author: Zahra Hashemizadeh, PhD, Prof Alborzi Clinical Microbiology Research Center, Shiraz University of Medical Sciences, Shiraz, IR Iran. Tel: +98-36474303, \\ Fax:+98-36474304, E-mail: zh.hashemiz@gmail.com
}

Received 2017 January 12; Revised 2017 February 18; Accepted 2017 February 25.

\begin{abstract}
Background: Voriconazole is a triazole antifungal agent with considerable inter and intra-individual variability in plasma concentrations. Therapeutic drug monitoring of voriconazole plays an important role in optimizing the efficacy and safety of the drug in patients.

Objectives: This study aimed at evaluating the performance of a simple agar well diffusion bioassay and comparing its utility with high-performance liquid chromatography (HPLC).

Methods: The clinical isolate of voriconazole hyper susceptible Candida kefyr was used for a simple agar well diffusion bioassay method. Acetonitrile precipitations followed by reverse-phase HPLC on C18 column, with ultra violet detection, were used for HPLC. Cross validation was done by evaluating the accuracy and precision of both methods in a large cohort of 180 samples from 60 voriconazole-treated patients.

Results: The validated bioassay method and HPLC, as a reference method, were found to be accurate and precise. A good correlation was found between the 2 methods with similar analytical range $(0.25-16 \mu \mathrm{g} / \mathrm{mL})$. The result of linear regression analysis revealed bioassay $=0.961(\mathrm{HPLC})+0.148 ; \mathrm{R}^{2}=0.965$; correlation coefficient $=0.982 ; \mathrm{n}=180$. Voriconazole serum concentration of patients ranged from $0.25 \mu \mathrm{g} / \mathrm{mL}$ to $5.41 \mu \mathrm{g} / \mathrm{mL}$ when using HPLC, and from $0.25 \mu \mathrm{g} / \mathrm{mL}$ to $5.71 \mu \mathrm{g} / \mathrm{mL}$ when using the bioassay method.

Conclusions: When laboratories are not equipped with HPLC, bioassay may be a reliable technique with sufficient accuracy and precision for monitoring voriconazole plasma concentration.
\end{abstract}

Keywords: Voriconazole, Drug Monitoring, Bioassay, Chromatography

\section{Background}

Invasive fungal infections are associated with high morbidity and mortality in patients with prolonged hospital stay, and recipients of hematopoietic stem cell and solid organ transplant $(1,2)$. Voriconazole is a wide-spectrum triazole medication recommended for the treatment of potentially life-threatening fungal infections $(3,4)$. It is considered as the therapy of choice for invasive aspergillosis (5), and a valid alternative for treating disseminated candidiasis (6) and other fungal infections caused by Fusarium and Scedosporium species $(7,8)$.

The mode of action for voriconazole is binding in the active-site cavity of cytochrome P450 sterol $14 \alpha$ demethylase enzyme and inhibiting the synthesis of ergosterol, cell-membrane, and fungal growth (9). The oral bioavailability, binding rate to plasma proteins, and tissue penetration of this agent are $>90 \%, 58 \%$, and 2 to $4.6 \mathrm{~L} / \mathrm{kg}$, respectively. The elimination half-life of voriconazole is approximately 6 hours with $<2 \%$ of the drug excreted unchanged in the urine (10). Voriconazole plasma levels are highly variable, attributed to several factors, which influ- ence its steady-state blood concentration $(11,12)$.

Genetic polymorphisms of cytochrome P450, mainly CYP2C19 enzyme, which effect the metabolism of voriconazole, patients' age, drug-drug interaction, specific clinical conditions such as organ failures and liver function abnormalities, lead to substantial inter and intra-individual variability of voriconazole plasma trough concentrations in clinical practice (13-15). These variations might be associated with decreased efficacy or increased toxicity (16). Low voriconazole levels $(<1.0 \mu \mathrm{g} / \mathrm{mL})$ are associated with therapeutic failure and elevated levels ( $\geq 5.5 \mu \mathrm{g} / \mathrm{mL}$ ) are correlated with an increased risk of adverse events (hepatotoxicity, hallucination, visual disturbance, and skin rash)(17-19).

Therapeutic drug monitoring (TDM) is a potentially useful tool for preventing toxicities related to voriconazole by clinicians (20). Different methods such as gas chromatography, mass spectrometry, and ultra-performance liquid chromatography have been described for the evaluation of voriconazole concentrations (21). Many studies have applied high-performance liquid chromatography (HPLC) as an accurate technique, to quantify voricona- 
zole plasma levels with acceptable sensitivity $(22,23)$. However, given the high price of the equipment for HPLC, this method is not widely used for voriconazole measurement in routine clinical laboratories. Bioassay has also been used as a microbiologic technique, which is reliable and easy to perform (24).

\section{Objectives}

This study aimed at describing a simple and valid agar well diffusion bioassay for quantification of voriconazole plasma levels and comparing the accuracy and precision of this method with HPLC in a group of voriconazole-treated patients.

\section{Methods}

\subsection{Preparation of Standards and Control Samples}

Reference standard of voriconazole (purity $\geq 98 \%$ ) was purchased from Sigma (Sigma-Aldrich, Germany). Two separate stock solutions were prepared by dissolving voriconazole in dimethyl sulfoxide (DMSO; Sigma-Aldrich, Germany) to obtain the concentration of the drug (1600 $\mu \mathrm{g} / \mathrm{mL})$. Calibration standard samples were prepared by mixing $990 \mu \mathrm{L}$ of human serum (Commercial human male AB Plasma, Sigma-Aldrich) with $10 \mu \mathrm{L}$ aliquots of the first stock solution in a series of 2 -fold dilutions to give the final concentrations of 16, 8.0, 4.0, 2.0, 1.0, 0.5, 0.25 , and $0.125 \mu \mathrm{g} / \mathrm{mL}$. Quality control samples, containing $0.25,0.5,1.0,2.0,4.0$, and $8.0 \mu \mathrm{g} / \mathrm{mL}$ of voriconazole were prepared in the same way using the second stock solution. For evaluating the selectivity and specificity of methods, other antifungals and antibacterial drugs consisting of fluconazole, itraconazole, caspofungin (Sigma-Aldrich), imipenem, amoxicillin/clavulanic acid, ciprofloxacin, ceftazidime, and gentamicin (Oxoid SA, Madrid, Spain) were used (25).

3.2. Clinical Performance and Concordance Between HighPerformance Liquid Chromatography and Bioassay

Clinical utility of bioassay was evaluated by comparing the measure of voriconazole plasma levels in 180 samples from 60 patients receiving voriconazole treatment, using both HPLC and bioassay methods. For oral administration, loading dose of $400 \mathrm{mg}$ on the first day, followed by 200 mg twice daily, for patients with weight of $\geq 40 \mathrm{~kg}$, and $200 \mathrm{mg}$ followed by $100 \mathrm{mg}$ twice daily for patients with weight of $\leq 40 \mathrm{~kg}$ were prescribed. For intravenous therapy, 2 loading doses of $6 \mathrm{mg} / \mathrm{kg} / 12$-hours on the first day, followed by $4 \mathrm{mg} / \mathrm{kg} / 12$-hours were used for all patients, according to the specific guideline (26). The patients receiving combination antifungal therapy were excluded from the study. Blood samples (3 mL EDTA) were drawn 30 minutes prior to administration of the next voriconazole dose (20). Plasma was stored at $-70^{\circ} \mathrm{C}$ for further analysis.

\subsection{Bioassay of Voriconazole}

The biological activity of voriconazole in serum samples was measured by a diffusion assay. A clinically isolated voriconazole susceptible strain (MIC $\leq 0.015$ $\mu \mathrm{g} / \mathrm{mL}$ ) of Candida kefyr, identified by API 20C AUX system (bioMerieux Vitek, Hazelwood, Mo.) and restriction fragment length polymorphism, was used as a test organism. The organism was cultured on sabouraud dextrose agar (MERCK, Germany) and colonies were suspended in $3.0 \mathrm{~mL}$ of sterile water. The turbidity was adjusted to $1 \mathrm{McF}$ arland standard equivalent to 1 - $5 \times 10^{8} \mathrm{CFU} / \mathrm{mL}$ (optical density at $530 \mathrm{~nm}$ between 0.23 and 0.27). A broth medium containing 6.7 g yeast nitrogen base (YNB, Difco Becton Dickinson), 30 g glucose (Sigma-Aldrich Chemie, Germany), and $5.9 \mathrm{~g}$ trisodium citrate (Sigma-Aldrich Chemie, Germany) was prepared in $1000 \mathrm{~mL}$ distilled water, and $\mathrm{pH}$ was adjusted to 7.0, and the solution was filtered by a syringe filter (Jet Biofil, China).

The agar medium (MERCK, Germany) was made (15 g/L) and autoclaved for 15 minutes at $121^{\circ} \mathrm{C}$. After cooling down to $48^{\circ} \mathrm{C}, 45 \mathrm{~mL}$ of agar, $5 \mathrm{~mL}$ of filtered broth medium, and $1 \mathrm{~mL}$ of adjusted C. kefyr suspension were gently mixed by inversion and poured in sterile a $150 \mathrm{~mm} \times 150 \mathrm{~mm}$ petri dish. The agar was left to solidify at room temperature for 30 to 45 minutes. In addition, 6 round wells $(5 \mathrm{~mm}$ diameter) were bored using sterile crock borer (Figure 1). Twenty-five microliters of each standard, control, and patients' plasma were pipetted to each well of the plate, allowed to diffuse through the agar at room temperature for 30 minutes, and incubated at $37^{\circ} \mathrm{C}$ for 24 hours. The inhibition zones were quantified by measuring the zone diameter using a metric caliper micrometer (Glattbach, Germany) with precision of $0.1 \mathrm{~mm}$. Measurements were done in duplicates by 2 different investigators. All test runs were repeated 3 times daily on 3 different days.

\subsection{High-Performance Liquid Chromatography Assay of Voriconazole}

\subsubsection{Sample Preparation}

Serial dilutions of calibration standard and quality control in human serum and serum samples of patients were processed as follows: $200 \mu \mathrm{L}$ ice-cold acetonitrile (Merck, Darmstadt, Germany) was added to the $200 \mu \mathrm{L}$ aliquots of each sample for protein precipitation. These 
Figure 1. Bioassay Plate for Voriconazole

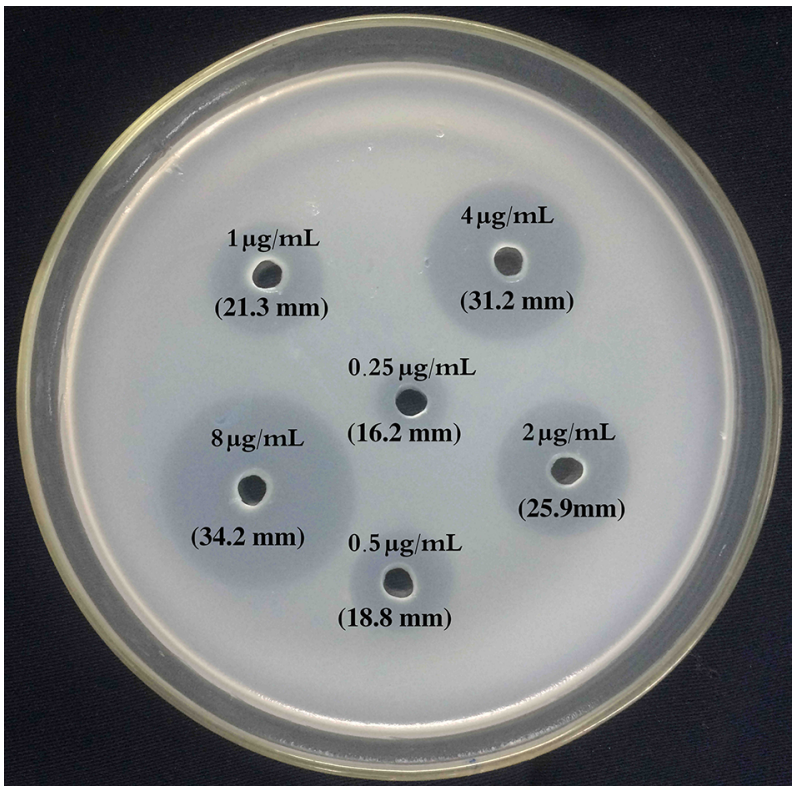

Numbers above the wells represent voriconazole concentrations and the numbers below the wells are the mean diameter of inhibition zones.

mixtures were vortex-mixed for 30 seconds and centrifuged at $13,800 \mathrm{rpm}$ and $25^{\circ} \mathrm{C}$ for 15 minutes. Supernatants were transferred to injection vials. For each run, $60 \mu \mathrm{L}$ of supernatant was injected to liquid chromatography (27).

\subsubsection{Chromatographic Conditions}

The HPLC method was described by Cendejas-Bueno et al. (27). Reversed- phase (RP-18) HPLC analyses were performed using a Knauer analytical HPLC with a K-1001 pump (Knauer, Berlin, Germany) and a variable wavelength ultraviolet spectrophotometric detector (Knauer PDA 2800) set at wavelength of $262 \mathrm{~nm}$. The separation was carried out by a $125-\mathrm{mm} \times 4.60-\mathrm{mm}$ inside-diameter reverse-phase column (Nucleodur 100-5 C18 ec), maintained at room temperature $\left(25^{\circ} \mathrm{C}\right)$. The mobile phase consisted of deionized water (Millipore Milli-Q system, Watford, UK) and HPLCgrade acetonitrile ( $\geq 99.9 \%$, Merck, Darmstadt, Germany) at $60 / 40(\mathrm{vol} / \mathrm{vol})$ proportions. The total run time was 9 minutes at a flow rate of $0.4 \mathrm{~mL} /$ minute. The EZChrom Elite Software was used to control the HPLC system, monitor the output signals, and plot the chromatograms.
3.5. Validation Procedure for Bioassay and High-Performance Liquid Chromatography

\subsubsection{Linearity Assessments}

To evaluate the linearity of the bioassay using 8 calibration standard samples (ranging from 0.125 to $16.0 \mu \mathrm{g} / \mathrm{mL}$ ), a standard curve for the concentrations of voriconazole $(\mu \mathrm{g} / \mathrm{mL})$ versus zones of inhibition diameter $(\mathrm{mm})$ was plotted. For HPLC, the standard curve was constructed by plotting the peak areas measured by HPLC against the concentrations of the 8 standards. Linearity assessments for both methods were performed by linear regression analysis and were validated with a correlation coefficient of (R2) $\geq 0.99$.

\subsubsection{Accuracy and Precision}

The inter-run and intra-run accuracy and precision (expressed as relative error and coefficient of variation, respectively) of HPLC and bioassay were determined by analyzing 6 quality control samples (0.25, 0.5, 1.0, 2.0, 4.0, and $8.0 \mu \mathrm{g} / \mathrm{mL}$ ). For the intra-run assay (within-day), the voriconazole concentrations in quality control samples were measured in triplicates on the same day and for the inter-run assay (between-day), quality control samples were processed during 3 non-consecutive days. The accuracy should have been within $\pm 15 \%$ deviation from the nominal values, precision within $\pm 15 \%$ of coefficient of variation, and the lower limit of quantification, should not have exceeded $20 \%$ of the coefficient of variation, as recommended by the U.S. food and drug administration (FDA) guide lines (28).

\subsubsection{Analytical Recovery}

Percentage recovery of voriconazole from plasma was calculated by comparing the peak area of 4 extracted serum samples spiked with voriconazole $(0.5,1.0,2.0$, and $4.0 \mu \mathrm{g} / \mathrm{mL}$ ) with 4 aqueous solutions of voriconazole of the same concentrations using the following formula: measured concentration in plasma/measured concentration in water $\times 100(28)$.

\subsubsection{Selectivity and Specificity}

Selectivity was tested using 6 independent batches of human plasma samples in order to check for the presence of potential interferences of endogenous substances. Specificity was indicated by the absence of interference peaks at same retention times of voriconazole for HPLC and no zone of inhibition in bioassay results. The specificity of both methods was determined by analyzing various blank samples and samples from patients, who had received commonly used anti-bacterials (imipenem, amoxicillin/clavulanic acid, ciprofloxacin, ceftazidime, and gen- 
tamicin) or antifungals (fluconazole, itraconazole, and caspofungin).

\subsection{Ethical Consideration}

This study was carried out in accordance with the guidelines of the declaration of Helsinki, as revised in Edinburgh (1975). The study protocol was approved by the ethics committee of Prof. Alborzi clinical microbiology research center (ec-92-6884). Written informed consents were obtained from all patients prior to the blood sampling.

\subsection{Statistical Analysis}

For calculations, HPLC was considered as the reference method. Linearity assessment for both methods (HPLC and bioassay) was performed by linear regression analysis. The within and between-run variability of the assays were estimated by computing the coefficient of variation and relative error. The agreement between both analytical methods was evaluated using the correlation coefficient.

\section{Results}

\subsection{Bioassay}

Regression analysis showed that the standard curve of the bioassay was logarithmic in the range of 0.25 to 16 $\mu \mathrm{g} / \mathrm{mL}$ with a correlation coefficient of $\mathrm{R}^{2}=0.989$ in all runs (Figures 1 and 2). The limit of detection and lower limit of quantification of the bioassay method were found to be $0.25 \mu \mathrm{g} / \mathrm{mL}$. The results of the intra and inter-run validation are shown in Table 1. The bioassay method was found to be precise, as the repeated measurements of the control samples (three times daily for three different days) showed the within and between-day accuracies of bioassay method ranged from $1.28 \%$ to $9.6 \%$ and $2.23 \%$ to $10 \%$, and the precisions ranged from $2.08 \%$ to $7.25 \%$ and $3.65 \%$ to $6.99 \%$, respectively. The coefficient of variation and relative error data were in agreement with international recommendations for bio-analytical methods (28).

\subsection{High Performance Liquid Chromatography}

High-performance liquid chromatography results demonstrated a linear relationship between peak height ratios and voriconazole concentrations over a range of 0.25 to $16 \mu \mathrm{g} / \mathrm{mL}\left(\mathrm{R}^{2}>0.99\right)$. The limit of detection and the lower limit of quantification by the HPLC method were found to be 0.125 and $0.25 \mu \mathrm{g} / \mathrm{mL}$, respectively. The mean retention time for voriconazole was $4.5 \pm 0.3$ minutes. The within and between-day accuracies of HPLC ranged from $-0.37 \%$ to $6 \%$ and $-3.2 \%$ to $4.95 \%$, while the precisions ranged from $0.33 \%$ to $6.65 \%$ and $0.6 \%$ to $4.59 \%$, respectively (Table

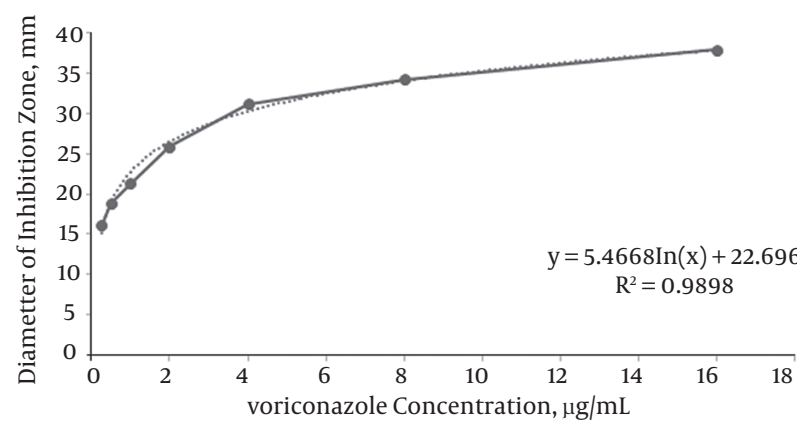

Figure 2. Standard Curve Linearity of Bioassay for Determining Voriconazole Concentrations in the Range of 0.25 to $16.0 \mu \mathrm{g} / \mathrm{mL}$

1). The percentage recovery of voriconazole from extracted samples was in the range of $94.7 \%$ to $100 \%$, indicating the consistent, precise, and reproducible extraction efficiency of the HPLC as the standard method. Endogenous components of voriconazole-free samples (blank) did not show any interference with peak heights of voriconazole at the retention times. Due to high specificity of the method, no interaction was observed between voriconazole signal, and possible co-administered of antifungal and antibacterial drugs.

\subsection{Correlation of Bioassay Results with High-Performance Liq-} uid Chromatography

The correlation between voriconazole levels measured by bioassay and HPLC in clinical samples was evaluated in 180 samples from 60 patients (Figure 3). Voriconazole serum concentration of patients ranged from $0.25 \mu \mathrm{g} / \mathrm{mL}$ to $5.41 \mu \mathrm{g} / \mathrm{mL}$ when using HPLC, and from $0.25 \mu \mathrm{g} / \mathrm{mL}$ to $5.71 \mu \mathrm{g} / \mathrm{mL}$ when using the bioassay method. Values below the lower limit of quantification were considered as $0.00 \mu \mathrm{g} / \mathrm{mL}$. Comparing the results of 2 analytical methods, $31 \%$ of the samples showed voriconazole concentration less than $0.25 \mu \mathrm{g} / \mathrm{mL}$ by HPLC and bioassay methods. The concordance between the results of HPLC and bioassay methodologies was assessed, as indicated by the scatterplot in Figure 3. The result of linear regression analysis showed bioassay $=0.961($ HPLC $)+0.148 ; \mathrm{R}^{2}=0.965$; correlation coefficient $=0.982 ; \mathrm{n}=180$.

\section{Discussion}

The rate of invasive fungal infections is growing parallel with increasing number of immunocompromised patients $(29,30)$. Voriconazole, as an effective agent, is currently used for the treatment of invasive aspergillosis (31). Despite initiating an appropriate drug and regimen, inadequate drug exposure at the site of infection might occur 
Table 1. Within-Day and Between-Day Variability of Voriconazole Concentration in Quality Control Samples by High-Performance Liquid Chromatography Method and Bioassay ${ }^{\mathrm{a}, \mathrm{b}}$

\begin{tabular}{|c|c|c|c|c|c|c|}
\hline \multirow{2}{*}{$\begin{array}{l}\text { Voriconazole Concentration of QC Samples, } \\
\mu \mathrm{g} / \mathbf{m L}\end{array}$} & \multicolumn{3}{|c|}{ Within-Day Variability } & \multicolumn{3}{|c|}{ Between-Day Variability } \\
\hline & $\mathbf{C}, \mu \mathrm{g} / \mathbf{m L}$ & Accuracy RE, \% & Precision CV, \% & $\mathbf{C}, \mu \mathrm{g} / \mathbf{m L}$ & Accuracy RE, \% & Precision CV, \% \\
\hline \multicolumn{7}{|l|}{ HPLC } \\
\hline 0.25 & $0.265 \pm 0.013$ & 6.00 & 4.90 & $0.261 \pm 0.012$ & 4.40 & 4.59 \\
\hline 0.5 & $0.526 \pm 0.035$ & 5.20 & 6.65 & $0.512 \pm 0.011$ & 2.40 & 2.14 \\
\hline 1.0 & $0.943 \pm 0.039$ & -5.70 & 4.13 & $0.968 \pm 0.030$ & -3.20 & 3.51 \\
\hline 2.0 & $2.088 \pm 0.087$ & 4.40 & 3.02 & $2.099 \pm 0.015$ & 4.95 & 0.71 \\
\hline 4.0 & $4.060 \pm 0.080$ & 1.50 & 1.97 & $4.090 \pm 0.033$ & 2.25 & 0.80 \\
\hline 8.0 & $7.970 \pm 0.264$ & -0.37 & 0.33 & $8.040 \pm 0.049$ & 0.50 & 0.60 \\
\hline \multicolumn{7}{|l|}{ Bioassay } \\
\hline 0.5 & $0.538 \pm 0.039$ & 7.60 & 7.25 & $0.543 \pm 0.038$ & 8.60 & 6.99 \\
\hline 1.0 & $1.030 \pm 0.055$ & 3.00 & 5.34 & $1.042 \pm 0.061$ & 4.20 & 5.85 \\
\hline 2.0 & $2.042 \pm 0.113$ & 2.10 & 5.53 & $2.080 \pm 0.093$ & 4.00 & 4.47 \\
\hline 4.0 & $4.193 \pm 0.234$ & 4.82 & 5.58 & $4.257 \pm 0.185$ & 6.42 & 4.34 \\
\hline 8.0 & $8.103 \pm 0.169$ & 1.28 & 2.08 & $8.179 \pm 0.299$ & 2.23 & 3.65 \\
\hline
\end{tabular}

Abbreviations: C, experimental concentration; CV\%: coefficient of variation in percent; HPLC, high-performance liquid chromatography; QC, quality control; RE\%: relative error in percent; SD, standard deviation.

${ }^{\mathrm{a}}$ Three times daily for three different days.

${ }^{\mathrm{b}}$ Values are expressed as mean $\pm \mathrm{SD}$.

due to pharmacokinetic variability of voriconazole (19). Therefore, to improve the efficacy and safety and minimize the risk of adverse events, therapeutic drug monitoring by an accurate and reliable assay is crucial. In this study, a validated simple bioassay method was compared with HPLC for the quantification of voriconazole levels in patients. Linear regression analysis showed an excellent correlation between the 2 analytical methods (correlation coefficient $=0.982$ ).

Based on FDA recommendations, the bioassay has valid criteria for measurement of voriconazole concentrations in biological matrices (28). This method gives the possibility to evaluate potency and monitor the biological activity of voriconazole in patients (32). Voriconazole level in plasma, following the administration of multiple oral or intravenous doses, varies from 1.0 to $5.5 \mu \mathrm{g} / \mathrm{mL}(20,33)$, which are within the limits in which the bioassay method was linear $(0.25-16 \mu \mathrm{g} / \mathrm{mL})$ in this study. Therefore, linearity range obtained by this method effectively covers what is currently believed to be the clinically relevant range for voriconazole concentrations in plasma. Therefore, there was not any limitation of practical applicability for the detection of voriconazole-related toxicity or therapeutic failure by the suggested bioassay method.
In this study, HPLC, as the standard method, was highly specific and no interaction was observed between voriconazole signal and possible co-administered antifungal and antibacterial drugs. One of the practical advantages of the proposed bioassay is being easy to perform with no special equipment and expertise required that might not be available in all clinical microbiology laboratories. Protein precipitation in samples was not used as a pretreatment procedure. Another advantage is a small volume of plasma sample needed to perform this bioassay (75 $\mu \mathrm{L}$ ), which is particularly important in the case of pediatric patients, compared to the HPLC method, which uses at least $200 \mu \mathrm{L}$ of plasma.

The proposed bioassay is slightly different from other microbiological methods described in previous studies (34-36). In this study, a specific clinical isolate of C. kefyr (voriconazole-hyper susceptible strain, MIC $\leq 0.015$ $\mu \mathrm{g} / \mathrm{mL}$ ) was used as the test organism. Previous experiments involving bioanalytical methods have used some types of reference strains provided by American type culture collection $(24,34)$ or applied a mutant of C. albicans, constructed by targeted deletions of genes in their studies (36). According to the present study, use of in-house clinical isolates of C. kefyr susceptible to voriconazole, which 
Figure 3. Correlation Between Voriconazole Serum Concentrations Measured by the Bioassay and High-Performance Liquid Chromatography Method

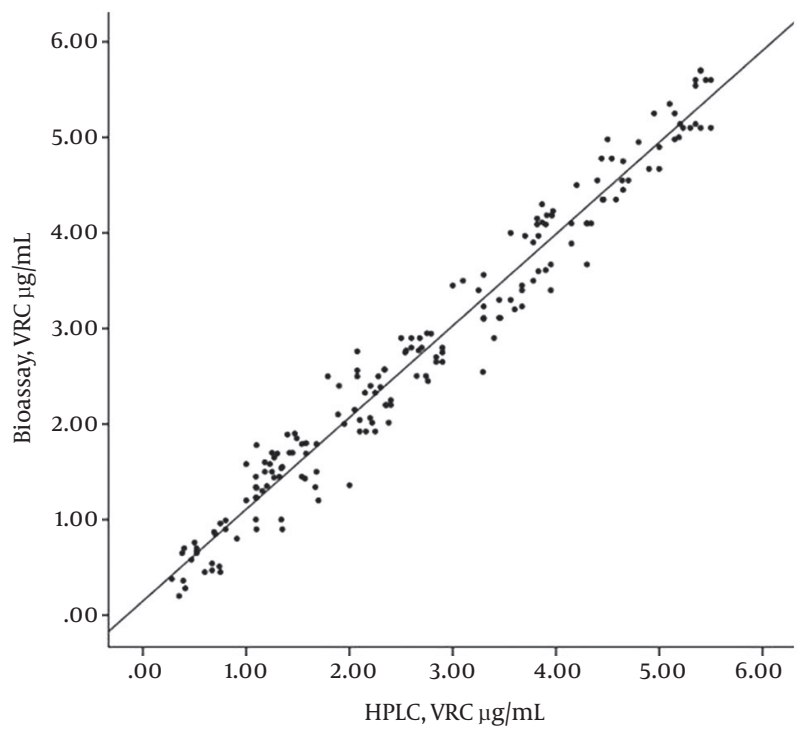

Linear regression result: bioassay $=0.961($ HPLC $)+0.148 ; \mathrm{R}^{2}=0.965 ;$ correlation coefficient $=0.982 ; \mathrm{n}=180$

provided well-defined and symmetric zones of growth inhibition, is suitable as the test organism. It is worth mentioning that the bioassay has some limitations.

Analytical methods such as HPLC exclusively rely on the assessment of compounds with a predefined chemical structure. It could discriminate the parent compound from related metabolites. The bioassay is unable to identify the active metabolites form of drugs. The other limitation of the bioassay method is combination of antifungal therapy by patients, which may effect the regular inhibition zone of the growth. Therefore, a clear interpretation of the voriconazole concentration is impossible in these patients. The third limitation is the time for conducting the test; the HPLC method is a valuable option when the results are needed quickly (3 hours), but in bioassay, results need longer analytical time, approximately 24 hours. Unfortunately, in many countries, HPLC equipment is very expensive and blood voriconazole concentrations are not evaluated for patients.

The current study evaluated the bioassay and HPLC method and revealed a good concordance between them for the measurements of voriconazole plasma levels in 180 samples from 60 patients. The proposed bioassay with sufficient accuracy and precision may be a valid alternative tool to HPLC in clinical laboratories without specialized facilities.

\section{Acknowledgments}

The authors thank $\mathrm{H}$. Khajehei, PhD, for copy editing of the manuscript. They express their special thanks to Dr Kamiar Zomorodian and Miss Froogh Karami for their help and cooperation in carrying out the lab procedure.

\section{Footnotes}

Authors' Contribution: Concept, design, data analysis, critical revision, and editing of the manuscript: Parisa Badiee; practical procedure, data analysis, and writing of the manuscript: Zahra Hashemizadeh; statistical analysis and critical revision the manuscript: Hashem Montaseri.

Funding/Support: This work was performed as part of Zahra Hashemizadeh's dissertation as a requirement for graduation as a PhD of Mycology from Prof. Alborzi clinical microbiology research center, Shiraz University of Medical Sciences, Shiraz, Iran. This project was financially supported by a grant ( 92 - 6884) from Shiraz University of Medical Sciences, Shiraz, Iran.

Conflict of Interest: All contributing authors declare that they had no financial or nonfinancial conflicts of interest related to the subject matter or materials discussed in the manuscript.

\section{References}

1. Pappas PG, Alexander BD, Andes DR, Hadley S, Kauffman CA, Freifeld A et al. Invasive fungal infections among organ transplant recipients: results of the Transplant-Associated Infection Surveillance Network (TRANSNET). Clin Infect Dis. 2010;50(8):1101-11. doi: 10.1086/651262. [PubMed: 20218876].

2. Badiee P, Alborzi A, Joukar M. Molecular assay to detect nosocomial fungal infections in intensive care units. Eur J Intern Med. 2011;22(6):611-5. doi: 10.1016/j.ejim.2011.08.025. [PubMed: 22075290].

3. Shang W, Feng G, Sun R, Wang X, Liu W, Zhang S, et al. Comparison of micafungin and voriconazole in the treatment of invasive fungal infections in kidney transplant recipients. J Clin Pharm Ther. 2012;37(6):652-6. doi: 10.1111/j.1365-2710.2012.01362.x. [PubMed: 22725946].

4. Badiee P, Alborzi A, Moeini M, Haddadi P, Farshad S, Japoni A, et al. Antifungal susceptibility of the aspergillus species by Etest and CLSI reference methods. Arch Iran Med. 2012;15(7):429-32. [PubMed: 22724880].

5. Dinser R, Grgic A, Kim YJ, Pfreundschuh M, Schubert J. Successful treatment of disseminated aspergillosis with the combination of voriconazole, caspofungin, granulocyte transfusions, and surgery followed by allogeneic blood stem cell transplantation in a patient with primary failure of an autologous stem cell graft. Eur J Haematol. 2005;74(5):438-41. doi: 10.1111/j.1600-0609.2004.00384.x. [PubMed: 15813919].

6. Kullberg BJ, Sobel JD, Ruhnke M, Pappas PG, Viscoli C, Rex JH, et al. Voriconazole versus a regimen of amphotericin $\mathrm{B}$ followed by fluconazole for candidaemia in non-neutropenic patients: a randomised non-inferiority trial. Lancet. 2005;366(9495):1435-42. doi: 10.1016/S0140-6736(05)67490-9. [PubMed: 16243088]. 
7. Stanzani M, Tumietto F, Vianelli N, Baccarani M. Update on the treatment of disseminated fusariosis: focus on voriconazole. Ther Clin Risk Manag. 2007;3(6):1165-73. [PubMed: 18516266].

8. Troke P, Aguirrebengoa K, Arteaga C, Ellis D, Heath CH, Lutsar I, et al. Treatment of scedosporiosis with voriconazole: clinical experience with 107 patients. Antimicrob Agents Chemother. 2008;52(5):1743-50. doi: 10.1128/AAC.01388-07. [PubMed: 18212110].

9. Xiao L, Madison V, Chau AS, Loebenberg D, Palermo RE, McNicholas PM. Three-dimensional models of wild-type and mutated forms of cytochrome P450 14alpha-sterol demethylases from Aspergillus fumigatus and Candida albicans provide insights into posaconazole binding. Antimicrob Agents Chemother. 2004;48(2):568-74. [PubMed: 14742211].

10. Theuretzbacher $U$, Ihle F, Derendorf $H$. Pharmacokinetic/pharmacodynamic profile of voriconazole. Clin Pharmacokinet. 2006;45(7):649-63. doi: 10.2165/00003088-200645070-00002. [PubMed: 16802848].

11. Denning DW, Ribaud P, Milpied N, Caillot D, Herbrecht R, Thiel E, et al. Efficacy and safety of voriconazole in the treatment of acute invasive aspergillosis. Clin Infect Dis. 2002;34(5):563-71. doi: 10.1086/324620. [PubMed: 11807679].

12. Badali H, Vaezi A, Aslani N, Zahedi N, Shiran MR. Overview of Voriconazole Pharmacokinetics and Pharmacodynamic Parameters. J Mazandaran Univ Med Sci. 2016;26(136):225-35.

13. Ikeda Y, Umemura K, Kondo K, Sekiguchi K, Miyoshi S, Nakashima M. Pharmacokinetics of voriconazole and cytochrome P450 2C19 genetic status. Clin Pharmacol Ther. 2004;75(6):587-8. doi: 10.1016/j.clpt.2004.02.002. [PubMed: 15179414].

14. Wang G, Lei HP, Li Z, Tan ZR, Guo D, Fan L, et al. The CYP2C19 ultra-rapid metabolizer genotype influences the pharmacokinetics of voriconazole in healthy male volunteers. EurJClin Pharmacol. 2009;65(3):281-5. doi: 10.1007/s00228-008-0574-7. [PubMed: 18982321].

15. Karlsson MO, Lutsar I, Milligan PA. Population pharmacokinetic analysis of voriconazole plasma concentration data from pediatric studies. Antimicrob Agents Chemother. 2009;53(3):935-44. doi: 10.1128/AAC.00751-08. [PubMed: 19075073].

16. Pakyz A, Bearman G. Adverse drug events complicate antifungal therapy for pulmonary aspergilloma. Consult Pharm. 2008;23(10):804-8. [PubMed: 19032016].

17. Job KM, Olson J, Stockmann C, Constance JE, Enioutina EY, Rower JE, et al. Pharmacodynamic studies of voriconazole: informing the clinical management of invasive fungal infections. Expert Rev Anti Infect Ther. 2016;14(8):731-46. doi: 10.1080/14787210.2016.1207526. [PubMed: 27355512].

18. Smith J, Andes D. Therapeutic drug monitoring of antifungals: pharmacokinetic and pharmacodynamic considerations. Therapeutic drug monitoring. LWW; 2008. pp. 167-72.

19. Agrawal AK, Sherman LK. Voriconazole-induced musical hallucinations. Infection. 2004;32(5):293-5. [PubMed: 15624894].

20. Pascual A, Calandra T, Bolay S, Buclin T, Bille J, Marchetti O. Voriconazole therapeutic drug monitoring in patients with invasive mycoses improves efficacy and safety outcomes. Clin Infect Dis. 2008;46(2):20111. doi: 10.1086/524669. [PubMed: 18171251].

21. Wang Z, Huang CK, Sun W, Xiao C, Wang ZS. UPLC-MS/MS determination of voriconazole in human plasma and its application to a pharmacokinetic study. Biomed Chromatogr. 2015;29(2):188-93. doi: 10.1002/bmc.3257. [PubMed: 24925071].

22. H Tang P. Quantification of Antifungal Drug Voriconazole in Serum and Plasma by HPLC-UV. Journal of Drug Metabolism \& Toxicology. 2013;04(02) doi: 10.4172/2157-7609.1000144.

23. Kahle K, Langmann P, Schirmer D, Lenker U, Keller D, Helle A, et al. Simultaneous determination of voriconazole and posaconazole concentrations in human plasma by high-performance liquid chromatography. Antimicrob Agents Chemother. 2009;53(7):3140-2. doi: 10.1128/AAC.00213-09. [PubMed: 19380591].

24. Perea S, Pennick GJ, Modak A, Fothergill AW, Sutton DA, Sheehan DJ, et al. Comparison of high-performance liquid chromatographic and microbiological methods for determination of voriconazole levels in plasma. Antimicrob Agents Chemother. 2000;44(5):1209-13. [PubMed: 10770753].

25. EMEA . Guideline on validation of bioanalytical methods EMEA; 2010. Available from: www.emea.europa.eu/docs/en-GB/doucmentlibrary/scientific-Guideline/2009/12/wc500018062.pdf.

26. EPAR-Product Information . Europen Medicines Agency (EMA) 2014. Available from: http://www.ema.europa.eu/docs/en_GB/ document_library/EPAR_Product_Information/human/002669/ WC500144015.pdf.

27. Cendejas-Bueno E, Rodriguez-Tudela JL, Cuenca-Estrella M, GomezLopez A. Development and validation of a fast HPLC/photodiode array detection method for the measurement of voriconazole in human serum samples. A reference laboratory experience. Enferm Infecc Microbiol Clin. 2013;31(1):23-8. doi: 10.1016/j.eimc.2012.03.003. [PubMed: 22776376].

28. Food and Drug Administration . FDA guidance for industry: bioanalytical method validation. Rockville: US Department of Health and Human Services; 2001.

29. Badiee P, Kordbacheh P, Alborzi A, Ramzi M, Shakiba E. Molecular detection of invasive aspergillosis in hematologic malignancies. Infection. 2008;36(6):580-4. doi: 10.1007/s15010-008-7385-8. [PubMed: 18854937].

30. Badiee P, Kordbacheh P, Alborzi A, Malekhoseini S, Zeini F, Mirhendi $\mathrm{H}$, et al. Prospective screening in liver transplant recipients by panfungal PCR-ELISA for early diagnosis of invasive fungal infections. Liver Transpl. 2007;13(7):1011-6. doi: 10.1002/lt.21175. [PubMed: 17600362].

31. Liu L, Zhou X, Wu T, Jiang H, Yang S, Zhang Y. Dose optimisation of voriconazole with therapeutic drug monitoring in children: a singlecentre experience in China. Int J Antimicrob Agents. 2017;49(4):483-7. doi: 10.1016/j.ijantimicag.2016.11.028. [PubMed: 28159657].

32. Gupta H, Aqil M, Khar RK, Ali A, Chander P. A single reversed-phase UPLC method for quantification of levofloxacin in aqueous humour and pharmaceutical dosage forms. J Chromatogr Sci. 2010;48(6):48490. [PubMed: 20822665].

33. Pascual A, Csajka C, Buclin T, Bolay S, Bille J, Calandra T, et al. Challenging recommended oral and intravenous voriconazole doses for improved efficacy and safety: population pharmacokinetics-based analysis of adult patients with invasive fungal infections. Clin Infect Dis. 2012;55(3):381-90. doi: 10.1093/cid/cis437. [PubMed: 22610925].

34. Cendejas-Bueno E, Cuenca-Estrella M, Gomez-Lopez A. Determination of voriconazole serum concentration by bioassay, a valid method for therapeutic drug monitoring for clinical laboratories. Antimicrob Agents Chemother. 2013;57(7):3437-40. doi: 10.1128/AAC.00323-13. [PubMed: 23650161].

35. Cendejas-Bueno E, Forastiero A, Rodriguez-Tudela JL, Cuenca-Estrella M, Gomez-Lopez A. HPLC/UV or bioassay: two valid methods for posaconazole quantification in human serum samples. Clin Microbiol Infect. 2012;18(12):1229-35. doi: 10.1111/j.1469-0691.2011.03732.x. [PubMed: 22192527].

36. Pascual A, Nieth V, Calandra T, Bille J, Bolay S, Decosterd LA, et al. Variability of voriconazole plasma levels measured by new highperformance liquid chromatography and bioassay methods. Antimicrob Agents Chemother. 2007;51(1):137-43. doi: 10.1128/AAC.00957-06. [PubMed: 17088483]. 